\title{
Screening the Cucumber Germplasm Collection for Fruit Storage Ability
}

\author{
Todd C. Wehner, ${ }^{1,2}$ Nischit V. Shetty ${ }^{3}$, and L. George Wilson ${ }^{1}$ \\ Department of Horticultural Science, North Carolina State University, Raleigh, \\ NC 27695-7609
}

\begin{abstract}
Additional index words. Cucumis sativus, postharvest physiology, vegetable breeding, storage life, shelf life, keeping ability
\end{abstract}

\begin{abstract}
All available cucumber (Cucumis sativus $\mathbf{L}$.) cultigens were tested for combining ability for fruit storage ability by crossing them with the gynoecious inbred Gy 14 . Fruit weight and firmness were measured before and after storage, and fruits were rated for water loss after storage. The cultigens with the lowest percentage of fruit weight loss during storage were PI 172839, PI 344067, PI 264667, PI 171612, PI 339245, PI 220171, PI 279469, and PI 368550; those with the lowest percentage of loss in fruit firmness were PI 379284, PI 339241, PI 414159, PI 422177, 'Regal', PI 109483, 'Addis', PI 285603, PI 257486, and 'Calypso'. The cultigens demonstrating the least fruit shriveling were 'Dasher II', 'Sprint 440', 'Texas Long', PI 390255, PI 432870, 'Pacer', PI 419078, PI 390247, PI 321011, and PI 414158. The 10 best cultigens from the initial screening study, along with the four worst cultigens and six checks, were retested directly (not as $F_{1}$ progeny) for fruit keeping ability in two storage conditions and at two harvest dates. No significant differences were detected between the two harvest dates and storage conditions.
\end{abstract}

The cucumber fruit is botanically classified as a pepo (an inferior berry) and is nonclimacteric (Biale and Young, 1981). Because cucumbers are often harvested and stored for several weeks before use by the consumer, keeping ability is an important trait. According to Buescher (1986), the fruits can be stored for 1 or 2 weeks under optimal conditions [i.e., $4^{\circ} \mathrm{C}, 100 \%$ relative humidity $(\mathrm{RH})$, and $1 \% \mathrm{O}_{2}$ and $30 \% \mathrm{CO}_{2}$ ] without loss of quality. Both environmental and genetic factors are involved in keeping ability; a large genetic component is the fruit type. Commercial types of cucumbers include american pickling (processing), european pickling, american slicing (fresh market), european greenhouse (parthenocarpic), oriental trellis (burpless), middleeastern cucumbers (Beit Alpha), and schalgurken (german pickling) (Wehner and Horton, 1986).

Cucumber breeders have developed cultivars that are white-spined rather than blackspined because of the linkage or pleiotropy between genes $B$ and $R$ (Hutchins, 1940). Large, immature fruits that would be green in


for publication 27 Sept. 1999. The use of trade names in this publication does not imply endorsement by the NCARS of the products named, nor criticism of similar ones not mentioned. Research funded in part by a grant from Claussen Pickle Co. We thank R.R. Horton, Jr., for his technical assistance. The cost of publishing this paper was defrayed in part by the payment of page charges. Under postal regulations, this paper therefore must be hereby marked advertisement solely to indicate this fact.

${ }^{1}$ Professor.

${ }^{2}$ To whom reprint requests should be addresed. E-mail address: todd_wehner@ncsu.edu

${ }^{3}$ Graduate Research Assistant.
}

the northern parts of the United States turn yellow to orange in the southern states if they have the $R$ gene. Therefore, cucumber breeders have developed white-spined cultivars since the 1940s to keep the fruits green longer (Wehner and Robinson, 1991).

Keeping ability in pickling cucumbers is critical and is related to the method of processing-brining (fermentation), freshpack (pasteurization), or cold-pack (refrigeration). Pickling cucumbers often are shipped from remote locations for fresh-pack operations; the time from harvest to processing usually lasts up to a week (Miller and Wehner, 1989). Fresh-pack processing of cucumbers has been widely accepted because of the high quality of the final product (attractive color and improved crispness). Refrigerated products are gaining in consumer popularity. Although they have a limited shelf life, they offer high quality. The perishable nature of cucumbers prevents the economical storage of fresh fruits for longer than 1 month. The advantages of brining over the other two pickling processes are that large quantities of fruits can be stored until needed for processing, and the finished product has a longer shelf life (Lower and Edwards, 1986).

The shelf life and pickling quality of cucumbers can be enhanced by spraying the plants with $100 \mathrm{mg} \cdot \mathrm{L}^{-1} \mathrm{~K}$ and $5000 \mathrm{mg} \cdot \mathrm{L}^{-1} \mathrm{Ca}$. Further improvement can result when fruits are packaged in perforated polyethylene bags and stored under refrigerated conditions $\left(5^{\circ} \mathrm{C}\right.$ and $85 \% \pm 5 \% \mathrm{RH})$. This reduces weight loss and fruit decay (Bakr and Gawish, 1993). Fruits treated with thiabenzadole [2-(4thiazolyl) benzimidazole] (a postharvest fungicide) and held in perforated polyethylene bags at room temperature for $9 \mathrm{~d}$ lost less weight, had less decay, and were firmer than nontreated controls (Shanan et al., 1978). Keep- ing quality of greenhouse-grown cucumbers was negatively influenced by certain energysaving measures (i.e., thermal screens and glasshouses with double roofing) (Janse and Welles, 1984). The removal of field heat immediately after harvest can enhance the postharvest fruit quality of produce (Kays, 1991), and hydrocooling of cucumbers has been recommended to enhance keeping ability. Navazio and Staub (1994) and Thomas and Staub (1992) reported that the most effective treatment was hydrocooling immediately after harvest followed by optimum storage conditions $\left(15^{\circ} \mathrm{C}\right.$ and $\left.85 \% \mathrm{RH}\right)$.

Optimum storage conditions rarely can be attained in the first few days after harvest. Improved keeping ability of cultivars held at ambient summer temperatures $\left(25-40{ }^{\circ} \mathrm{C}\right.$ in North Carolina) would help reduce postharvest losses of fresh-market and pickling cucumbers, and would improve the yield of greenstock purchased and shipped to consumers and processors.

The objective of this study was to evaluate the U.S. Dept. of Agriculture (USDA) cucumber germplasm collection, along with additional cultivars, for keeping ability using rapid methods of measurement. The "best" and "worst" cultigens (cultivars, breeding lines, and plant introduction accessions) identified in the germplasm collection screening study were further retested using more elaborate methods.

\section{Materials and Methods}

All experiments were performed at the Horticultural Crops Research Station at Clinton, N.C., using recommended horticultural practices (Hughes et al., 1983; Schultheis, 1990), and optimized field plot trials. Three replications of once-over harvest have been recommended for maximum efficiency to determine which cucumber lines have commercial potential for multiple-harvest trials (Wehner, 1986; Wehner and Miller, 1984). A plot size of $1.2 \times 1.5 \mathrm{~m}$ was optimum for yield evaluation for once-over harvest of pickling cucumbers (Swallow and Wehner, 1986). Wehner and Miller (1987) recommended the use of small single-row plots without end borders rather than large, multiple-row, bordered plots. Small-plot, single-harvest trials were more efficient than large-plot, multipleharvest trials, which require more time and labor(Wehner, 1986, 1989; Wehner and Miller, 1984). Swallow and Wehner (1989) reported that maximum information (1/variance) was obtained by allocating test plots of cucumber lines to different seasons and years instead of using different locations and replications. Field plots at Clinton were the best for yield evaluation in North Carolina (Wehner, 1987).

\section{Germplasm tested}

Germplasm screening study. A total of 756 cultigens from a total of 44 countries were evaluated in the experiment, including 15 checks. Most of the cultigens were plant introduction accessions; also included were 47 
breeding lines (old cultivars, current cultivars, or experimental inbreds and hybrids). Cultigens designated with aPInumber were obtained from the USDA germplasm collection at Ames, Iowa. Old cultivars were obtained from the National Seed Storage Laboratory (NSSL) in Fort Collins, Colo. Countries with the largest number of entries were Turkey, People's Republic of China, Iran, Yugoslavia, India, Japan, and Czechoslovakia. To make them gynoecious, all cultigens were crossed with Gy 14, a gynoecious pickling cucumber inbred, under controlled greenhouse conditions at the Horticultural Science Greenhouses at North Carolina State Univ., Raleigh; this assured fruit set under local field conditions. The cultigens designated "checks" were the cultigens per se (not the $F_{1}$ resulting from a cross with Gy 14). Cultigens with high combining ability for keeping quality should be good genetic sources for direct use in a breeding program. However, cultigens that performed poorly for combining ability might still be good sources for direct use if their superior keeping ability was due primarily to recessive genes. Recessive genes for keeping ability might not be expressed in the hybrid if Gy 14 had dominant genes at the loci involved. Gy 14 was intermediate (212 of 756) in performance for keeping ability, as indicated by loss in fruit weight. Some of the best and worst cultigens were retested as hybrids with Gy 14 and as the cultigen per se, so we were able to measure in part the importance of recessive genes in keeping ability.
Retest study. A total of 20 cultigens were tested for this study. Cultigens in the top $10 \%$ for fruit weight loss from the germplasm screening study (Gy 14 x PI220171, Gy 14 x PI279469, Gy 14 x PI321006, Gy x PI211962, Gy 14 x Producer, Gy 14 x PI422177, Regal, PI 220171, Producer, and PI 422177) were chosen to represent cultigens for good keeping ability. Four cultigens in the worst $10 \%$ range for fruit loss in the screening study (Wautoma, Gy 14 x PI339244, Gy 14 x PI255934, and PI 339244) were chosen to represent cultigens with the worst keeping ability. Six standard commercial checks were added to the study ('Sumter', 'Wisconsin SMR 18', 'Calypso', 'Poinsett 76', 'Marketmore 76', and 'Dasher II').
Table 1. Keeping ability of fruits of 756 cultigens tested at Clinton, N.C. ${ }^{z}$

\begin{tabular}{|c|c|c|c|c|}
\hline Cultigen & Seed source $y$ & Wt loss (\%) & $\begin{array}{c}\text { Firm. } \\
\text { loss }(\%)\end{array}$ & Shriveling ${ }^{x}$ \\
\hline PI 172839 & Turkey & $-52^{\mathrm{w}}$ & 21 & 6.7 \\
\hline PI 344067 & Turkey & $-24^{\mathrm{w}}$ & 19 & 7.3 \\
\hline PI 264667 & Germany & $-11^{\mathrm{w}}$ & 11 & 6.3 \\
\hline PI 171612 & Turkey & $-2^{\mathrm{w}}$ & 6 & 7.0 \\
\hline PI 339245 & Turkey & 3 & 24 & 6.0 \\
\hline PI 220171 & Afghanistan & 8 & 14 & 4.3 \\
\hline PI 279469 & Japan & 9 & 16 & 5.7 \\
\hline PI 368550 & Yugoslavia & 9 & 24 & 7.7 \\
\hline PI 263047 & USSR & 10 & 14 & 6.3 \\
\hline PI 326597 & Hungary & 10 & 13 & 6.7 \\
\hline PI 343452 & USSR & 10 & 16 & 7.0 \\
\hline Check-Gy 5 & Check & 10 & 14 & 5.0 \\
\hline PI 264230 & France & 10 & 6 & 6.0 \\
\hline PI 344439 & Iran & 11 & 14 & 5.7 \\
\hline PI 277741 & Netherlands & 11 & 11 & 5.7 \\
\hline PI 167197 & Turkey & 11 & 14 & 6.0 \\
\hline PI 182192 & Turkey & 11 & $-3^{\mathrm{w}}$ & 5.0 \\
\hline PI 263083 & P.R. China & 11 & 1 & 6.0 \\
\hline PI 419079 & P.R. China & 11 & 10 & 5.3 \\
\hline PI 175696 & Turkey & 12 & 13 & 7.0 \\
\hline Ansansky & NSSL & 12 & 22 & 6.0 \\
\hline PI 357851 & Yugoslavia & 12 & 13 & 6.3 \\
\hline PI 392292 & USSR & 12 & 16 & 5.7 \\
\hline PI 137836 & Iran & 12 & 14 & 6.3 \\
\hline PI 432894 & P.R. China & 12 & 16 & 5.0 \\
\hline PI 321006 & Taiwan & 12 & 10 & 4.7 \\
\hline PI 432895 & P.R. China & 12 & 13 & 5.0 \\
\hline PI 164950 & Turkey & 12 & 9 & 6.0 \\
\hline PI 211962 & Iran & 12 & 25 & 4.3 \\
\hline Producer & NSSL & 12 & 23 & 6.7 \\
\hline PI 172848 & Turkey & 12 & 16 & 6.0 \\
\hline PI 288993 & Hungary & 12 & 12 & 5.7 \\
\hline PI 376063 & Israel & 12 & 7 & 5.7 \\
\hline PI 204568 & Turkey & 12 & 11 & 5.3 \\
\hline PI 288995 & Hungary & 12 & 12 & 5.3 \\
\hline PI 343451 & USSR & 12 & $-1^{\mathrm{w}}$ & 6.3 \\
\hline PI 357862 & Yugoslavia & 12 & 15 & 7.0 \\
\hline PI 351139 & USSR & 12 & 7 & 6.7 \\
\hline PI 137839 & Iran & 12 & 6 & 5.7 \\
\hline PI 257487 & P.R. China & 12 & 20 & 6.0 \\
\hline PI 326594 & Hungary & 12 & 12 & 5.7 \\
\hline PI 264666 & Germany & 12 & 17 & 6.3 \\
\hline PI 422177 & Czechoslovakia & 12 & $-6^{\mathrm{w}}$ & 4.5 \\
\hline Check-Regal & Check & 13 & $-6^{\mathrm{w}}$ & 4.3 \\
\hline PI 175691 & Turkey & 13 & 17 & 6.0 \\
\hline PI 288994 & Hungary & 13 & 21 & 5.7 \\
\hline PI 288996 & Hungary & 13 & 6 & 5.3 \\
\hline PI 344349 & Turkey & 13 & 11 & 7.0 \\
\hline PI 209065 & U.S.-Ohio & 13 & 14 & 6.0 \\
\hline PI 222243 & Iran & 13 & 8 & 6.3 \\
\hline PI 209068 & U.S.-Ohio & 13 & 9 & 6.3 \\
\hline PI 267747 & U.S.-Oklahoma & 13 & 12 & 6.7 \\
\hline PI 172849 & Turkey & 13 & 18 & 5.7 \\
\hline
\end{tabular}

\begin{tabular}{|c|c|c|c|c|}
\hline Cultigen & Seed source ${ }^{y}$ & Wt loss (\%) & $\begin{array}{c}\text { Firm. } \\
\text { loss }(\%)\end{array}$ & Shriveling $^{x}$ \\
\hline PI 422191 & Czechoslovakia & 13 & $-1^{\mathrm{w}}$ & 5.3 \\
\hline PI 137856 & Iran & 13 & 4 & 5.7 \\
\hline PI 355053 & Iran & 13 & 0 & 5.3 \\
\hline PI 171606 & Turkey & 13 & 12 & 6.0 \\
\hline PR 27 & NSSL & 13 & 14 & 5.3 \\
\hline PI 264665 & Germany & 13 & 11 & 6.0 \\
\hline PI 422198 & Czechoslovakia & 13 & 14 & 6.5 \\
\hline PI 169387 & Turkey & 13 & 18 & 6.0 \\
\hline PI 324239 & Sweden & 13 & 11 & 5.7 \\
\hline PI 279464 & Japan & 13 & 27 & 5.0 \\
\hline PI 390954 & USSR & 13 & 4 & 6.3 \\
\hline PI 205995 & Sweden & 13 & 18 & 7.3 \\
\hline Check-Gy 4 & Check & 13 & 18 & 4.7 \\
\hline PI 234517 & U.S.-S.C. & 13 & 5 & 4.0 \\
\hline PI 314425 & USSR & 13 & 6 & 5.7 \\
\hline PI 197086 & India & 13 & 30 & 7.0 \\
\hline PI 280096 & USSR & 13 & 13 & 6.3 \\
\hline PI 432888 & P.R. China & 13 & 28 & 5.0 \\
\hline Check-Gy 2 & Check & 13 & 8 & 5.0 \\
\hline PI 321009 & Taiwan & 13 & 15 & 5.3 \\
\hline PI 292012 & Israel & 13 & 13 & 6.0 \\
\hline PI 279465 & Japan & 13 & 11 & 5.7 \\
\hline PI 211982 & Iran & 13 & 15 & 6.3 \\
\hline PI 267197 & P.R. China & 14 & 2 & 5.0 \\
\hline PI 172851 & Turkey & 14 & 18 & 5.7 \\
\hline PI 164952 & Turkey & 14 & 9 & 6.3 \\
\hline PI 211985 & Iran & 14 & 7 & 5.3 \\
\hline PI 209067 & U.S.-Ohio & 14 & 15 & 5.0 \\
\hline PI 281448 & Korea & 14 & 8 & 5.0 \\
\hline WS Davis Perf. & NSSL & 14 & 5 & 5.0 \\
\hline PI 368554 & Yugoslavia & 14 & --- & 6.0 \\
\hline PI 419214 & Hong Kong & 14 & 15 & 4.7 \\
\hline PI 109481 & Turkey & 14 & 20 & 5.3 \\
\hline PI 248778 & Iran & 14 & 13 & 5.7 \\
\hline PI 212233 & Japan & 14 & 12 & 4.7 \\
\hline Shamrock R & NSSL & 14 & 20 & 5.3 \\
\hline PI 432870 & P.R. China & 14 & 22 & 3.0 \\
\hline PI 176951 & Turkey & 14 & 19 & 5.3 \\
\hline PI 368551 & Yugoslavia & 14 & 5 & 6.0 \\
\hline PI 176952 & Turkey & 14 & 10 & 7.0 \\
\hline PI 183224 & Egypt & 14 & 14 & 5.7 \\
\hline PI 357836 & Yugoslavia & 14 & 7 & 5.7 \\
\hline PI 263084 & P.R. China & 14 & 2 & 5.0 \\
\hline PI 302443 & P.R. China & 14 & 9 & 5.7 \\
\hline PI 357852 & Yugoslavia & 14 & 14 & 4.0 \\
\hline Check-Sprint 440 & Check & 14 & 4 & 3.0 \\
\hline Cubit & NSSL & 14 & 10 & 4.0 \\
\hline PI 308916 & USSR & 14 & 10 & 6.3 \\
\hline PI 196289 & India & 14 & 15 & 7.0 \\
\hline PI 285603 & Poland & 14 & $-5^{\mathrm{w}}$ & 4.7 \\
\hline PI 267088 & USSR & 14 & 12 & 7.0 \\
\hline PI 257494 & Iran & 14 & 23 & 6.7 \\
\hline PI 193497 & Ethiopia & 14 & 27 & 7.0 \\
\hline
\end{tabular}




\section{Cultural practices}

Germplasm screening study. Seeds were planted on raised, shaped beds $1.5 \mathrm{~m}$ apart. Plots were $1.2 \mathrm{~m}$ long and $1.5 \mathrm{~m}$ wide with 1.2-m alleys at each end. Fourteen seeds were planted in each plot on 12 May and the plants were thinned to a uniform stand of 10 plants per plot on 29 May 1987. No significant disease problems were observed. The guard rows were planted around the field, and at the end of each row. 'Sumter', a monoecious inbred, was planted in border rows and at the ends of each row (62 $\mathrm{m}$ long) to provide competition and pollen. Fertilizer was incorporated before planting at a rate of $90 \mathrm{~N}-39 \mathrm{P}-74 \mathrm{~K} \mathrm{~kg} \cdot \mathrm{ha}^{-1}$, with an additional 34 $\mathrm{kg} \cdot \mathrm{ha}^{-1} \mathrm{~N}$ applied at vine tip-over stage. A tank mix of $2.2 \mathrm{~kg} \cdot \mathrm{ha}^{-1}$ of naptalam $\{2-[(1-$ naphthalenylamino) carbonyl] benzoic acid $\}$ and $4.4 \mathrm{~kg} \cdot \mathrm{ha}^{-1}$ of bensulide $\{O, O$-bis $(1-$ methylethyl) $S$-[2-[(phenylsulfonyl) amino] ethyl] phosphorodithioate $\}$ was applied preplant for weed control. Irrigation was applied when needed for a total of $25-40 \mathrm{~mm}$ per week. Four fruits were harvested from each plot and evaluated for keeping ability following storage in brown paper bags at room temperature $\left(23 \pm 2{ }^{\circ} \mathrm{C}\right)$ and $55 \% \pm 7 \%$ $\mathrm{RH}$.

Retest study. Twenty cultigens identified during the initial screening study were planted in the spring season (Table 2). The plots $(2.8 \times 1.5 \mathrm{~m})$ were separated by $0.3-\mathrm{m}$ alleys at each end, and had 20 plants each. 'Sumter' was planted at each side of the field, and at each end of the row, to provide competition and pollen. Two harvest dates (1 and 13 July 1988) and two storage environments (an air-conditioned laboratory at $22 \pm$ $2{ }^{\circ} \mathrm{C}$ and $55 \% \pm 7 \% \mathrm{RH}$ and a storage building at $25 \pm 3{ }^{\circ} \mathrm{C}$ and $79 \% \pm 11 \% \mathrm{RH}$ without air conditioning) were used in the study. Fruit having a diameter of $40-50 \mathrm{~mm}$ were harvested from each plot and three were placed in each of five brown paper bags. Four of the bags were placed in either the laboratory or the storage building for 7 or $14 \mathrm{~d}$. Fruit in the fifth bag were measured immediately upon harvest and were designated the day 0 control.
Table 1. Continued.

\begin{tabular}{|c|c|c|c|c|}
\hline Cultigen & Seed source ${ }^{y}$ & Wt loss (\%) & $\begin{array}{c}\text { Firm. } \\
\text { loss }(\%)\end{array}$ & Shriveling $^{x}$ \\
\hline$\overline{\text { PI } 177361}$ & Turkey & 14 & 17 & 5.5 \\
\hline PI 169389 & Turkey & 14 & 0 & 7.0 \\
\hline PI 257486 & P.R. China & 14 & $-5^{\mathrm{w}}$ & 6.3 \\
\hline PI 338236 & Turkey & 14 & 20 & 5.0 \\
\hline PI 422181 & Czechoslovakia & 14 & 6 & 4.3 \\
\hline PI 222783 & Iran & 14 & 27 & 6.7 \\
\hline PI 113334 & P.R. China & 14 & 22 & 6.3 \\
\hline PI 092806 & P.R. China & 14 & 4 & 6.0 \\
\hline PI 218036 & Iran & 14 & 15 & 5.3 \\
\hline PI 226510 & Iran & 14 & 6 & 5.7 \\
\hline PI 176526 & Turkey & 14 & 6 & 6.0 \\
\hline PI 188749 & Egypt & 14 & 18 & 6.0 \\
\hline PI 432889 & P.R. China & 14 & 15 & 6.3 \\
\hline PI 326595 & Hungary & 14 & 16 & 6.0 \\
\hline PI 358814 & Malaysia & 14 & 24 & 6.7 \\
\hline PI 267086 & USSR & 14 & 26 & 7.0 \\
\hline PI 344435 & Iran & 14 & 13 & 6.0 \\
\hline PI 169398 & Turkey & 14 & 16 & 6.5 \\
\hline PI 451975 & Canada & 15 & 19 & 5.7 \\
\hline PI 114339 & Japan & 15 & 12 & 5.0 \\
\hline PI 169386 & Turkey & 15 & 18 & 6.3 \\
\hline York State Pickle & NSSL & 15 & 11 & 5.7 \\
\hline PI 432860 & P.R. China & 15 & 13 & 5.0 \\
\hline PI 370448 & Yugoslavia & 15 & 11 & 6.7 \\
\hline PI 183231 & Egypt & 15 & 21 & 5.3 \\
\hline PI 206954 & Turkey & 15 & 24 & 6.0 \\
\hline PI 285609 & Poland & 15 & 19 & 6.3 \\
\hline PI 283900 & Czechoslovakia & 15 & 11 & 6.7 \\
\hline PI 172845 & Turkey & 15 & 13 & 6.0 \\
\hline PI 419183 & P.R. China & 15 & 18 & 5.0 \\
\hline PI 137857 & Iran & 15 & 11 & 5.3 \\
\hline PI 422176 & Czechoslovakia & 15 & 2 & 5.0 \\
\hline PI 249561 & Thailand & 15 & 3 & 6.3 \\
\hline PI 183677 & Turkey & 15 & 23 & 6.0 \\
\hline PI 211589 & Afghanistan & 15 & 16 & 7.0 \\
\hline PI 419010 & P.R. China & 15 & 23 & 4.0 \\
\hline PI 172847 & Turkey & 15 & 13 & 6.0 \\
\hline PI 344437 & Iran & 15 & 19 & 5.3 \\
\hline PI 175679 & Turkey & 15 & 15 & 6.7 \\
\hline PI 174177 & Turkey & 15 & 22 & 5.7 \\
\hline Calypso & N.C. State Univ. & 15 & $-4^{w}$ & 4.7 \\
\hline PI 175695 & Turkey & 15 & 11 & 6.3 \\
\hline PI 200818 & Burma & 15 & 15 & 5.7 \\
\hline Tiny Dill & NSSL & 15 & 11 & 6.0 \\
\hline PI 167358 & Turkey & 15 & 8 & 5.7 \\
\hline PI 487424 & P.R. China & 15 & 14 & 5.0 \\
\hline Check-Dasher II & Check & 15 & 10 & 2.3 \\
\hline PI 288332 & India & 15 & 0 & 5.3 \\
\hline PI 339241 & Turkey & 15 & $-12^{\mathrm{w}}$ & 6.3 \\
\hline PI 285606 & Poland & 15 & 14 & 5.7 \\
\hline PI 368558 & Yugoslavia & 15 & 20 & 6.3 \\
\hline PI 344347 & Turkey & 15 & 4 & 6.7 \\
\hline PI 178887 & Turkey & 15 & 18 & 6.3 \\
\hline
\end{tabular}

Table 1. Continued.

\begin{tabular}{|c|c|c|c|c|}
\hline Cultigen & Seed source ${ }^{y}$ & Wt loss $(\%)$ & $\begin{array}{c}\text { Firm. } \\
\text { loss }(\%)\end{array}$ & Shriveling $^{x}$ \\
\hline PI 357850 & Yugoslavia & 15 & 11 & 6.3 \\
\hline PI 211728 & Afghanistan & 15 & 7 & 5.7 \\
\hline PI 229309 & Iran & 15 & 44 & 7.0 \\
\hline PI 209064 & U.S.-Ohio & 15 & 7 & 6.0 \\
\hline PI 356809 & USSR & 15 & 16 & 6.7 \\
\hline Check-Calypso & Check & 15 & 14 & 4.0 \\
\hline PI 174170 & Turkey & 15 & 4 & 6.0 \\
\hline Snows Pickling & NSSL & 15 & 18 & 4.3 \\
\hline PI 275411 & Netherlands & 15 & 9 & 6.3 \\
\hline PI 222099 & Afghanistan & 15 & 13 & 6.7 \\
\hline National Pickling & NSSL & 15 & 12 & 5.3 \\
\hline PI 169304 & Turkey & 15 & 21 & 6.7 \\
\hline PI 288237 & Egypt & 15 & 3 & 5.7 \\
\hline PI 227209 & Japan & 15 & 18 & 7.0 \\
\hline PI 227664 & Iran & 15 & 20 & 6.0 \\
\hline PI 283902 & Czechoslovakia & 15 & 25 & 6.3 \\
\hline Check-M 21 & Check & 15 & 13 & 4.0 \\
\hline PI 174173 & Turkey & 15 & 14 & 5.3 \\
\hline PI 321008 & Taiwan & 15 & 25 & 6.3 \\
\hline PI 172852 & Turkey & 15 & 13 & 6.3 \\
\hline PI 314426 & USSR & 15 & 15 & 6.0 \\
\hline PI 339247 & Turkey & 15 & 13 & 6.7 \\
\hline PI 264226 & France & 15 & 17 & 6.0 \\
\hline PI 279463 & Japan & 15 & 18 & 5.7 \\
\hline PI 344348 & Turkey & 15 & 12 & 6.7 \\
\hline PI 436609 & P.R. China & 15 & 6 & 6.0 \\
\hline PI 369717 & Poland & 15 & 27 & 6.5 \\
\hline PI 418964 & P.R. China & 15 & 15 & 4.0 \\
\hline PI 177360 & Turkey & 15 & 13 & 6.0 \\
\hline PI 390257 & Japan & 15 & 14 & 4.3 \\
\hline PI 419078 & P.R. China & 15 & 17 & 3.3 \\
\hline PI 172844 & Turkey & 15 & 5 & 6.0 \\
\hline PI 169315 & Turkey & 15 & 16 & 7.0 \\
\hline PI 414158 & U.S.-Hawaii & 15 & 9 & 3.7 \\
\hline PI 169397 & Turkey & 16 & 6 & 6.7 \\
\hline PI 211980 & Iran & 16 & 5 & 6.3 \\
\hline PI 288238 & Egypt & 16 & 26 & 5.7 \\
\hline M 21 & N.C. State Univ. & 16 & 5 & 4.3 \\
\hline PI 264668 & Germany & 16 & 5 & 6.0 \\
\hline PI 179921 & India & 16 & 13 & 7.0 \\
\hline PI 466922 & USSR & 16 & 7 & 4.7 \\
\hline PI 458853 & USSR & 16 & 0 & 6.5 \\
\hline PI 220790 & Afghanistan & 16 & 27 & 7.0 \\
\hline PI 105340 & P.R. China & 16 & 9 & 6.0 \\
\hline PI 211975 & Iran & 16 & 19 & 6.3 \\
\hline PI 419017 & P.R. China & 16 & 15 & 3.7 \\
\hline PI 321011 & Taiwan & 16 & 4 & 3.5 \\
\hline PI 344353 & Turkey & 16 & 7 & 7.7 \\
\hline PI 227208 & Japan & 16 & 23 & 3.7 \\
\hline PI 165499 & India & 16 & 3 & 7.0 \\
\hline PI 432893 & P.R. China & 16 & 19 & 6.0 \\
\hline PI 211978 & Iran & 16 & 15 & 4.7 \\
\hline Gy 14 & N.C. State Univ. & 16 & 29 & 4.5 \\
\hline
\end{tabular}


Breeding, Cultivars, Rootstocks, \& Germplasm Resources

Table 1. Continued.

\begin{tabular}{|c|c|c|c|c|}
\hline Cultigen & Seed source ${ }^{y}$ & Wt loss (\%) & $\begin{array}{c}\text { Firm. } \\
\text { loss }(\%)\end{array}$ & Shriveling $^{\mathrm{x}}$ \\
\hline PI 211117 & Israel & 16 & 23 & 4.7 \\
\hline PI 172838 & Turkey & 16 & 16 & 6.3 \\
\hline PI 175120 & India & 16 & 20 & 7.0 \\
\hline PI 172846 & Turkey & 16 & 3 & 6.3 \\
\hline PI 432883 & P.R. China & 16 & 7 & 4.7 \\
\hline PI 338234 & Turkey & 16 & 20 & 7.0 \\
\hline PI 458852 & USSR & 16 & 12 & 5.0 \\
\hline PI 478367 & P.R. China & 16 & 12 & 5.3 \\
\hline PI 233932 & Canada & 16 & 14 & 7.3 \\
\hline PI 135123 & New Zealand & 16 & $-2^{\mathrm{w}}$ & 7.3 \\
\hline PI 251519 & Iran & 16 & 26 & 7.0 \\
\hline PI 109483 & Turkey & 16 & $-6^{w}$ & 4.7 \\
\hline Texas Long & NSSL & 16 & 18 & 3.0 \\
\hline Clinton & N.C. State Univ. & 16 & 6 & 5.0 \\
\hline PI 211979 & Iran & 16 & 14 & 5.7 \\
\hline Model & NSSL & 16 & 9 & 5.3 \\
\hline PI 344351 & Turkey & 16 & 18 & 6.3 \\
\hline PI 176517 & Turkey & 16 & 4 & 6.3 \\
\hline Early Green Cluster & NSSL & 16 & 7 & 7.0 \\
\hline PI 357844 & Yugoslavia & 16 & 13 & 7.0 \\
\hline PI 432866 & P.R. China & 16 & 2 & 5.3 \\
\hline PI 103049 & P.R. China & 16 & 19 & 5.3 \\
\hline PI 344444 & Iran & 16 & 17 & 4.0 \\
\hline PI 211977 & Iran & 16 & $-4^{w}$ & 6.7 \\
\hline PI 174172 & Turkey & 16 & 6 & 5.7 \\
\hline PI 206425 & Turkey & 16 & 4 & 6.3 \\
\hline PI 171611 & Turkey & 16 & 25 & 7.0 \\
\hline PI 222985 & Iran & 16 & 15 & 5.7 \\
\hline PI 169402 & Turkey & 16 & 13 & 5.7 \\
\hline PI 257286 & Spain & 16 & 16 & 5.7 \\
\hline PI 344445 & Iran & 16 & 25 & 5.3 \\
\hline PI 344441 & Iran & 16 & 26 & 5.3 \\
\hline PI 175693 & Turkey & 16 & 18 & 6.0 \\
\hline PI 357859 & Yugoslavia & 16 & 14 & 7.0 \\
\hline PI 432850 & P.R. China & 16 & 21 & 6.3 \\
\hline PI 264664 & Germany & 16 & 13 & 5.7 \\
\hline PI 271753 & Netherlands & 16 & 14 & 5.0 \\
\hline PI 432892 & P.R. China & 16 & 21 & 5.7 \\
\hline PI 390255 & Japan & 16 & 20 & 3.0 \\
\hline PI 171605 & Turkey & 16 & 5 & 7.0 \\
\hline PI 269480 & West Pakistan & 16 & 17 & 6.3 \\
\hline PI 342950 & Denmark & 16 & 15 & 6.7 \\
\hline Early Fortune & NSSL & 16 & 5 & 6.3 \\
\hline PI 432867 & P.R. China & 16 & 25 & 5.7 \\
\hline PI 169392 & Turkey & 16 & 23 & 6.0 \\
\hline PI 326598 & Hungary & 16 & 2 & 5.7 \\
\hline PI 292011 & Israel & 16 & 28 & 5.7 \\
\hline Sprint 440 & Asgrow & 17 & 17 & 4.7 \\
\hline PI 172843 & Turkey & 17 & 29 & 7.3 \\
\hline PI 137853 & Iran & 17 & 12 & 5.7 \\
\hline PI 264229 & France & 17 & 22 & 4.0 \\
\hline Delcrow & NSSL & 17 & 14 & 4.5 \\
\hline PI 401733 & Puerto Rico & 17 & 10 & 4.0 \\
\hline PI 179676 & India & 17 & 21 & 4.3 \\
\hline PI 436610 & P.R. China & 17 & 20 & 4.3 \\
\hline PI 379282 & Yugoslavia & 17 & 11 & 8.0 \\
\hline PI 427090 & P.R. China & 17 & 25 & 5.0 \\
\hline PI 137848 & Iran & 17 & 20 & 6.7 \\
\hline PI 357845 & Yugoslavia & 17 & 10 & 6.3 \\
\hline PI 432879 & P.R. China & 17 & 19 & 6.0 \\
\hline PI 435946 & USSR & 17 & 16 & 5.7 \\
\hline PI 167134 & Turkey & 17 & 5 & 6.0 \\
\hline PI 478365 & P.R. China & 17 & 17 & 4.7 \\
\hline PI 432865 & P.R. China & 17 & 8 & 4.3 \\
\hline PI 354952 & Denmark & 17 & 12 & 6.0 \\
\hline PI 357841 & Yugoslavia & 17 & 8 & 6.3 \\
\hline PI 175686 & Turkey & 17 & 26 & 6.7 \\
\hline PI 263082 & P.R. China & 17 & 20 & 5.7 \\
\hline PI 401734 & Puerto Rico & 17 & 11 & 4.3 \\
\hline PI 109484 & Turkey & 17 & 2 & 6.0 \\
\hline PI 418963 & P.R. China & 17 & 11 & 4.3 \\
\hline PI 204692 & Turkey & 17 & 19 & 4.7 \\
\hline PI 167079 & Turkey & 17 & 18 & 5.0 \\
\hline PI 285604 & Poland & 17 & 14 & 4.7 \\
\hline PI 293432 & Lebanon & 17 & 19 & 6.3 \\
\hline
\end{tabular}

Table 1. Continued.

\begin{tabular}{|c|c|c|c|c|}
\hline Cultigen & Seed source ${ }^{y}$ & Wt loss $(\%)$ & $\begin{array}{c}\text { Firm. } \\
\text { loss }(\%)\end{array}$ & Shriveling $^{\mathrm{x}}$ \\
\hline PI 288992 & Hungary & 17 & 16 & 5.3 \\
\hline PI 285605 & Poland & 17 & 37 & 6.7 \\
\hline PI 171604 & Turkey & 17 & 9 & 6.7 \\
\hline PI 419108 & P.R. China & 17 & 23 & 5.0 \\
\hline PI 283899 & Czechoslovakia & 17 & 15 & 5.7 \\
\hline PI 226461 & Iran & 17 & 17 & 6.3 \\
\hline PI 169353 & Turkey & 17 & 18 & 6.0 \\
\hline PI 357865 & Yugoslavia & 17 & 7 & 6.0 \\
\hline PI 422186 & Czechoslovakia & 17 & 11 & 5.0 \\
\hline PI 432853 & P.R. China & 17 & 14 & 4.3 \\
\hline PI 182190 & Turkey & 17 & 14 & 6.0 \\
\hline PI 169383 & Turkey & 17 & 17 & 7.0 \\
\hline PI 390246 & Japan & 17 & 18 & 4.0 \\
\hline PI 246930 & Afghanistan & 17 & 11 & 5.3 \\
\hline PI 167050 & Turkey & 17 & 8 & 4.5 \\
\hline PI 489754 & P.R. China & 17 & 27 & 4.0 \\
\hline PI 390951 & USSR & 17 & 60 & 7.5 \\
\hline PI 357860 & Yugoslavia & 17 & 11 & 6.3 \\
\hline PI 172841 & Turkey & 17 & 5 & 5.7 \\
\hline Boston Pickling & NSSL & 17 & 17 & 6.7 \\
\hline PI 222987 & Iran & 17 & 10 & 6.3 \\
\hline PI 263080 & USSR & 17 & 13 & 6.7 \\
\hline PI 326596 & Hungary & 17 & 19 & 5.3 \\
\hline PI 357837 & Yugoslavia & 17 & 9 & 6.7 \\
\hline Arlington WS & NSSL & 17 & 21 & 5.0 \\
\hline PI 181756 & Lebanon & 17 & 6 & 5.7 \\
\hline PI 171600 & Turkey & 17 & 14 & 7.0 \\
\hline PI 379278 & Yugoslavia & 17 & 5 & 6.3 \\
\hline PI 169381 & Turkey & 17 & 19 & 6.3 \\
\hline PI 422168 & Czechoslovakia & 17 & 14 & 4.7 \\
\hline PI 458849 & USSR & 17 & 16 & 6.3 \\
\hline PI 204690 & Turkey & 17 & 11 & 5.7 \\
\hline PI 357839 & Yugoslavia & 18 & 11 & 5.3 \\
\hline PI 422185 & Czechoslovakia & 18 & 9 & 5.3 \\
\hline PR 39 & NSSL & 18 & 16 & 4.7 \\
\hline PI 321007 & Taiwan & 18 & 23 & 5.7 \\
\hline PI 192940 & P.R. China & 18 & 19 & 6.3 \\
\hline PI 226509 & Iran & 18 & 21 & 6.7 \\
\hline PI 390247 & Japan & 18 & 31 & 3.3 \\
\hline PI 344432 & Iran & 18 & 13 & 6.3 \\
\hline PI 355055 & Iran & 18 & 4 & 6.7 \\
\hline PI 356833 & GBE & 18 & 26 & 4.3 \\
\hline PI 175690 & Turkey & 18 & 9 & 5.7 \\
\hline PI 176957 & Turkey & 18 & 6 & 6.0 \\
\hline PI 177363 & Syria & 18 & 14 & 5.7 \\
\hline PI 275412 & Netherlands & 18 & 21 & 5.3 \\
\hline PI 167052 & Turkey & 18 & 14 & 6.7 \\
\hline Favor II & NSSL & 18 & 11 & 6.7 \\
\hline PI 432859 & P.R. China & 18 & 12 & 4.7 \\
\hline PI 169391 & Turkey & 18 & 7 & 6.3 \\
\hline PI 296387 & Iran & 18 & 15 & 7.0 \\
\hline PI 261608 & Spain & 18 & 8 & 5.0 \\
\hline PI 169401 & Turkey & 18 & 0 & 6.7 \\
\hline PI 368549 & Yugoslavia & 18 & 18 & 7.0 \\
\hline PI 181910 & Syria & 18 & 20 & 5.3 \\
\hline PI 267743 & P.R. China & 18 & 12 & 5.0 \\
\hline PI 227210 & Japan & 18 & 26 & 5.3 \\
\hline PI 182188 & Turkey & 18 & 20 & 5.7 \\
\hline PI 357831 & Yugoslavia & 18 & 21 & 7.0 \\
\hline PI 357868 & Yugoslavia & 18 & 17 & 5.7 \\
\hline PI 344352 & Turkey & 18 & 1 & 6.7 \\
\hline PI 212599 & Afghanistan & 18 & 13 & 7.0 \\
\hline PI 174167 & Turkey & 18 & 20 & 5.0 \\
\hline PI 181940 & Syria & 18 & 23 & 4.7 \\
\hline PI 390256 & Japan & 18 & 14 & 6.0 \\
\hline PI 197087 & India & 18 & 18 & 6.7 \\
\hline PI 178884 & Turkey & 18 & 31 & 7.3 \\
\hline PI 222782 & Iran & 18 & 25 & 6.0 \\
\hline PI 249550 & Iran & 18 & 15 & 5.7 \\
\hline PI 137835 & Iran & 18 & 21 & 6.0 \\
\hline PI 218199 & Lebanon & 18 & 25 & 7.0 \\
\hline PI 344438 & Iran & 18 & 15 & 6.0 \\
\hline PI 179260 & Turkey & 18 & 21 & 6.0 \\
\hline PI 172840 & Turkey & 18 & 18 & 5.0 \\
\hline PI 172842 & Turkey & 18 & 24 & 6.3 \\
\hline
\end{tabular}




\section{Traits measured}

Germplasm screening study. Data were collected on: fruit weight loss (\%), firmness loss (\%), and a visual rating for appearance (fruit shriveling). Fruit weight was measured before storage and after 2 weeks, when the fruits were rated for shriveling and firmness. Shriveling was rated subjectively on a $0-9$ scale with $0=$ none (no appearance of shriveling of fruit skin), $1-3=$ slight, $4-6=$ moderate, and 7-9 $=$ severe (skin very shriveled). Firmness was the amount of force $(\mathrm{N})$ required to penetrate the exocarp (skin) and mesocarp (flesh) with an 8-mm-diameter tester head (McCormick Fruit Tech, Yakima, Wash.) on the top carpel of the blossom end of the fruit.
Retest study. Data were recorded 0,7, and 14 $\mathrm{d}$ after harvest. The traits measured were fresh weight, firmness, percentage of shriveling, degree of yellowing, incidence of postharvest diseases, fresh taste, seedcell wateriness, placental hollows, and carpel separation.

Quality traits (taste, seedcell, placental hollows, and carpel separation) were rated subjectively on a $1-9$ scale $(1-3=$ poor, $4-6=$ intermediate, $7-9=$ excellent). Taste ratings of 1-3 indicated a bad flavor or bitter taste, 4 6 a slightly off-flavor, and 7-9 a good flavor and sweet taste. Seedcell wateriness was rated based on the degree to which the firm, crisp seedcell became soft and watery; ratings of 13 indicated that the seedcell was very watery, and a rating of 7-9 indicated that it was firm and crisp. Placental hollows rating was based on the length of placental hollows in the fruit. A rating of 1-3 indicated two or three hollows running most of the length of the fruits, 4-6 indicated one or two placental hollows running less than the entire length of the fruit, and 7-9 indicated that there was no defect (or no more than one placental hollow running less than half the length of the fruit). Carpel separation was rated based on the degree of carpel separation. A rating of 1-3 indicated carpels separated, and the center of the fruit hollow, 46 indicated carpels obviously separated, and 7-9 indicated that there was no defect (or carpels slightly but not obviously separated). Average quality, damage index, percentage loss in fresh fruit weight, and percentage of
Table 1. Continued.

\begin{tabular}{|c|c|c|c|c|}
\hline Cultigen & Seed source ${ }^{y}$ & Wt loss $(\%)$ & $\begin{array}{c}\text { Firm. } \\
\text { loss }(\%)\end{array}$ & Shriveling $^{\mathrm{x}}$ \\
\hline PI 176956 & Turkey & 18 & 12 & 5.7 \\
\hline Straight 8 & NSSL & 18 & 23 & 4.3 \\
\hline PI 109482 & Turkey & 18 & 13 & 7.0 \\
\hline PI 306785 & Canada & 18 & 22 & 3.7 \\
\hline PI 274902 & Great Britain & 18 & 10 & 5.7 \\
\hline PI 249562 & Thailand & 18 & 15 & 4.0 \\
\hline PI 163217 & India & 18 & 5 & 6.3 \\
\hline PI 357867 & Yugoslavia & 18 & 9 & 6.0 \\
\hline Everbearing & NSSL & 18 & 2 & 5.7 \\
\hline PI 227207 & Japan & 18 & 22 & 5.0 \\
\hline PI 135122 & New Zealand & 18 & 40 & 7.0 \\
\hline PI 174164 & Turkey & 18 & 30 & 6.7 \\
\hline PI 478364 & P.R. China & 18 & 9 & 5.0 \\
\hline PI 414159 & U.S.-Hawaii & 18 & $-8^{w}$ & 4.7 \\
\hline PI 458848 & USSR & 18 & 6 & 7.0 \\
\hline PI 262990 & Netherlands & 18 & 16 & 6.7 \\
\hline PI 137846 & Iran & 18 & 8 & 6.0 \\
\hline PI 379285 & Yugoslavia & 18 & 8 & 5.3 \\
\hline PI 390265 & Japan & 18 & 38 & 7.0 \\
\hline PI 251520 & Iran & 18 & 18 & 7.3 \\
\hline PI 178886 & Turkey & 18 & 19 & 6.0 \\
\hline PI 379287 & Yugoslavia & 18 & 2 & 6.7 \\
\hline PI 271331 & India & 19 & 19 & 7.0 \\
\hline PI 169403 & Turkey & 19 & 17 & 5.7 \\
\hline PI 271754 & Netherlands & 19 & 15 & 6.0 \\
\hline PI 432857 & P.R. China & 19 & 47 & 5.7 \\
\hline PI 379284 & Yugoslavia & 19 & $-13^{\mathrm{w}}$ & 5.0 \\
\hline PI 400270 & Japan & 19 & 24 & 7.0 \\
\hline PI 223437 & Afghanistan & 19 & 7 & 6.3 \\
\hline PI 165506 & India & 19 & 22 & 7.0 \\
\hline PI 458855 & USSR & 19 & 6 & 6.5 \\
\hline PI 206952 & Turkey & 19 & 18 & 6.0 \\
\hline PI 169388 & Turkey & 19 & 22 & 6.3 \\
\hline PI 489752 & P.R. China & 19 & 20 & 5.7 \\
\hline PI 422172 & Czechoslovakia & 19 & 18 & 4.7 \\
\hline PI 390953 & USSR & 19 & 10 & 7.0 \\
\hline PI 175683 & Turkey & 19 & 6 & 6.3 \\
\hline PI 171601 & Turkey & 19 & 22 & 5.7 \\
\hline PI 308915 & USSR & 19 & 18 & 6.7 \\
\hline PI 355052 & Israel & 19 & 8 & 6.0 \\
\hline PI 368548 & Yugoslavia & 19 & 16 & 6.7 \\
\hline PI 357857 & Yugoslavia & 19 & 23 & 7.0 \\
\hline PI 339246 & Turkey & 19 & 19 & 7.0 \\
\hline PI 179259 & Turkey & 19 & 6 & 6.0 \\
\hline PI 267087 & USSR & 19 & 6 & 6.3 \\
\hline PI 176516 & Turkey & 19 & 16 & 6.0 \\
\hline PI 482464 & Zimbabwe & 19 & 22 & 4.0 \\
\hline PI 255936 & Netherlands & 19 & 34 & 6.7 \\
\hline PI 165029 & Turkey & 19 & 19 & 6.7 \\
\hline PI 175680 & Turkey & 19 & 9 & 7.0 \\
\hline PI 171602 & Turkey & 19 & 22 & 6.3 \\
\hline PI 267942 & Japan & 19 & 11 & 6.7 \\
\hline
\end{tabular}

Table 1. Continued.

\begin{tabular}{|c|c|c|c|c|}
\hline Cultigen & Seed source ${ }^{y}$ & Wt loss $(\%)$ & $\begin{array}{c}\text { Firm. } \\
\text { loss }(\%)\end{array}$ & Shriveling $^{\mathrm{x}}$ \\
\hline PI 267746 & India & 19 & 7 & 7.0 \\
\hline PI 183445 & India & 19 & 23 & 6.7 \\
\hline PI 220789 & Afghanistan & 19 & 16 & 5.7 \\
\hline Shogoin & NSSL & 19 & 27 & 5.7 \\
\hline PI 304803 & U.S.-New York & 19 & 21 & 5.3 \\
\hline PI 357854 & Yugoslavia & 19 & 9 & 6.5 \\
\hline PI 169380 & Turkey & 19 & 14 & 6.3 \\
\hline PI 181753 & Syria & 19 & 14 & 5.7 \\
\hline PI 175694 & Turkey & 19 & 27 & 6.3 \\
\hline PI 106063 & P.R. China & 19 & 7 & 6.3 \\
\hline PI 391569 & P.R. China & 20 & 11 & 5.5 \\
\hline PI 344350 & Turkey & 20 & 27 & 6.7 \\
\hline PI 356832 & Netherlands & 20 & 3 & 5.3 \\
\hline PI 285607 & Poland & 20 & 3 & 6.7 \\
\hline PI 344433 & Iran & 20 & 13 & 5.7 \\
\hline PI 179678 & India & 20 & 12 & 4.7 \\
\hline PI 339250 & Turkey & 20 & 25 & 6.7 \\
\hline PI 177359 & Turkey & 20 & 23 & 4.7 \\
\hline PI 173889 & India & 20 & 20 & 5.7 \\
\hline PI 436673 & P.R. China & 20 & 31 & 4.7 \\
\hline Check-WI 2757 & Check & 20 & --- & 4.0 \\
\hline PI 390952 & USSR & 20 & 11 & 5.3 \\
\hline PI 164734 & India & 20 & 1 & 6.7 \\
\hline PI 401732 & Puerto Rico & 20 & 26 & 5.7 \\
\hline PI 422180 & Czechoslovakia & 20 & 31 & 4.5 \\
\hline PI 489753 & P.R. China & 20 & 18 & 5.7 \\
\hline PI 211988 & Iran & 20 & 8 & 5.7 \\
\hline PI 209069 & U.S.-Ohio & 20 & 24 & 6.3 \\
\hline PI 271334 & India & 20 & 18 & 6.7 \\
\hline PI 436648 & P.R. China & 20 & 26 & 5.0 \\
\hline PI 422196 & Czechoslovakia & 20 & 30 & 4.0 \\
\hline PI 458847 & USSR & 20 & 14 & 5.7 \\
\hline PI 269482 & West Pakistan & 20 & 14 & 6.3 \\
\hline PI 292010 & Israel & 20 & 8 & 5.0 \\
\hline PI 165046 & Turkey & 20 & 24 & 6.5 \\
\hline PI 135345 & Afghanistan & 20 & 21 & 6.3 \\
\hline PI 217644 & India & 20 & 29 & 7.3 \\
\hline PI 321010 & Taiwan & 20 & 13 & 6.7 \\
\hline PI 262974 & India & 20 & 24 & 6.3 \\
\hline PI 435947 & USSR & 20 & 19 & 6.3 \\
\hline PI 267935 & Japan & 20 & 16 & 7.0 \\
\hline PI 458850 & USSR & 20 & 6 & 7.0 \\
\hline PI 169394 & Turkey & 20 & 19 & 5.7 \\
\hline PI 390259 & Japan & 20 & 7 & 6.3 \\
\hline PI 171610 & Turkey & 20 & 19 & 7.0 \\
\hline PI 418989 & P.R. China & 20 & 8 & 7.5 \\
\hline PI 169351 & Turkey & 20 & 27 & 7.7 \\
\hline PI 390252 & Japan & 20 & 18 & 6.7 \\
\hline PI 175111 & India & 20 & 1 & 6.0 \\
\hline PI 263081 & P.R. China & 21 & 26 & 6.7 \\
\hline PI 211967 & Iran & 21 & 9 & 6.7 \\
\hline PI 319216 & Un. Arab Repub. & 21 & 11 & 6.3 \\
\hline
\end{tabular}


Breeding, Cultivars, Rootstocks, \& Germplasm Resources

Table 1. Continued.

\begin{tabular}{|c|c|c|c|c|}
\hline Cultigen & Seed source ${ }^{y}$ & Wt loss (\%) & $\begin{array}{c}\text { Firm. } \\
\text { loss }(\%)\end{array}$ & Shriveling $^{x}$ \\
\hline PI 338235 & Turkey & 21 & 8 & 6.0 \\
\hline PI 179263 & Turkey & 21 & 31 & 7.0 \\
\hline PI 169396 & Turkey & 21 & 17 & 6.7 \\
\hline PI 164819 & India & 21 & 20 & 7.7 \\
\hline PI 169393 & Turkey & 21 & 12 & 6.7 \\
\hline PI 212059 & Greece & 21 & 15 & 5.3 \\
\hline PI 390258 & Japan & 21 & 46 & 6.7 \\
\hline PI 357849 & Yugoslavia & 21 & 14 & 7.0 \\
\hline PI 422190 & Czechoslovakia & 21 & 21 & 4.3 \\
\hline PI 250147 & W. Pakistan & 21 & 8 & 6.7 \\
\hline PI 263049 & USSR & 21 & 17 & 6.3 \\
\hline PI 357835 & Yugoslavia & 21 & 3 & 6.5 \\
\hline PI 163214 & India & 21 & 23 & 7.3 \\
\hline PI 263079 & USSR & 21 & 26 & 4.7 \\
\hline PI 174166 & Turkey & 21 & 33 & 7.0 \\
\hline PI 169400 & Turkey & 21 & 11 & 6.7 \\
\hline PI 176954 & Turkey & 21 & 34 & 7.0 \\
\hline PI 164670 & India & 21 & 11 & 5.0 \\
\hline PI 206043 & U.S.-Puerto Rico & 21 & 37 & 6.7 \\
\hline PI 418962 & P.R. China & 21 & 27 & 5.0 \\
\hline PI 137847 & Iran & 21 & 45 & 7.7 \\
\hline PI 422170 & Czechoslovakia & 21 & 28 & 5.3 \\
\hline PI 178888 & Turkey & 21 & 29 & 7.3 \\
\hline PI 419136 & P.R. China & 21 & 16 & 5.3 \\
\hline PI 432890 & P.R. China & 21 & 19 & 5.3 \\
\hline PI 357840 & Yugoslavia & 21 & 31 & 7.0 \\
\hline PI 209066 & U.S.-Ohio & 21 & 29 & 7.0 \\
\hline PI 263048 & USSR & 21 & 16 & 7.0 \\
\hline PI 176953 & Turkey & 21 & 45 & 7.0 \\
\hline PI 267943 & Japan & 21 & 23 & 7.3 \\
\hline PI 419009 & P.R. China & 21 & 11 & 4.7 \\
\hline Muronium & NSSL & 21 & 57 & 7.0 \\
\hline PI 171609 & Turkey & 21 & 36 & 6.7 \\
\hline PI 390262 & Japan & 21 & 34 & 4.5 \\
\hline PI 357847 & Yugoslavia & 21 & 25 & 5.0 \\
\hline Polaris & NSSL & 21 & 41 & 5.5 \\
\hline PI 306180 & Poland & 21 & 17 & 6.0 \\
\hline Check-Addis & Check & 21 & $-6^{w}$ & 6.0 \\
\hline PI 483342 & Korea & 21 & 24 & 5.0 \\
\hline PI 221440 & Afghanistan & 22 & 12 & 6.0 \\
\hline PI 390269 & Japan & 22 & 15 & 7.0 \\
\hline PI 370449 & Yugoslavia & 22 & 18 & 8.0 \\
\hline PI 344434 & Iran & 22 & 17 & 6.0 \\
\hline White Wonder & NSSL & 22 & 3 & 6.3 \\
\hline PI 422188 & Czechoslovakia & 22 & 31 & 6.0 \\
\hline Pacer & Harris-Moran & 22 & -- & 3.0 \\
\hline PI 181874 & Syria & 22 & 20 & 6.0 \\
\hline PI 206953 & Turkey & 22 & 18 & 7.0 \\
\hline Long of Keschmet & NSSL & 22 & 27 & 7.0 \\
\hline PI 344440 & Iran & 22 & 12 & 5.7 \\
\hline PI 432873 & P.R. China & 22 & 38 & 6.3 \\
\hline PI 390253 & Japan & 22 & 20 & 7.0 \\
\hline PI 137844 & Iran & 22 & 18 & 7.5 \\
\hline PI 422173 & Czechoslovakia & 22 & 10 & 6.0 \\
\hline PI 167389 & Turkey & 22 & 36 & 7.0 \\
\hline PI 391568 & P.R. China & 22 & 26 & 6.7 \\
\hline PI 173892 & India & 22 & 28 & 6.0 \\
\hline PI 344443 & Iran & 22 & 15 & 6.7 \\
\hline PI 357863 & Yugoslavia & 22 & 16 & 6.0 \\
\hline PI 169385 & Turkey & 22 & 27 & 7.7 \\
\hline PI 207476 & Afghanistan & 22 & 19 & 5.3 \\
\hline PI 304805 & U.S.-New York & 22 & 17 & 6.5 \\
\hline PSI & NSSL & 22 & 20 & 4.3 \\
\hline PI 174174 & Turkey & 22 & 18 & 6.3 \\
\hline PI 173674 & Turkey & 22 & 35 & 7.3 \\
\hline PI 391573 & P.R. China & 22 & 33 & 5.0 \\
\hline PI 368556 & Yugoslavia & 22 & 36 & 7.7 \\
\hline PI 289698 & Australia & 22 & 5 & 6.3 \\
\hline PI 211984 & Iran & 22 & 12 & 5.0 \\
\hline PI 211943 & Iran & 22 & 14 & 6.3 \\
\hline PI 109275 & Turkey & 22 & 18 & 6.7 \\
\hline PI 414157 & U.S.-Oregon & 22 & 20 & 6.7 \\
\hline PI 360939 & Netherlands & 22 & 15 & 6.7 \\
\hline PI 279468 & Japan & 23 & 24 & 7.0 \\
\hline PI 193496 & Ethiopia & 23 & 13 & 6.7 \\
\hline
\end{tabular}

Table 1. Continued.

\begin{tabular}{|c|c|c|c|c|}
\hline Cultigen & Seed source ${ }^{y}$ & Wt loss $(\%)$ & $\begin{array}{c}\text { Firm. } \\
\text { loss }(\%)\end{array}$ & Shriveling $^{\mathrm{x}}$ \\
\hline PI 164816 & India & 23 & 2 & 7.7 \\
\hline PI 181942 & Syria & 23 & 24 & 5.3 \\
\hline PI 296121 & Egypt & 23 & 12 & 7.0 \\
\hline PI 293923 & U.S.-S.C. & 23 & 35 & 7.0 \\
\hline Check-Sumter & Check & 23 & 20 & 6.7 \\
\hline PI 264231 & France & 23 & 22 & 7.3 \\
\hline PI 390266 & Japan & 23 & 24 & 6.5 \\
\hline PI 182189 & Turkey & 23 & 13 & 5.7 \\
\hline PI 163213 & India & 23 & 22 & 6.0 \\
\hline PI 390248 & Japan & 23 & 31 & 7.5 \\
\hline PI 176924 & Turkey & 23 & 32 & 7.7 \\
\hline PI 357834 & Yugoslavia & 23 & 29 & 7.0 \\
\hline PI 390240 & Japan & 23 & 34 & 8.0 \\
\hline PI 181752 & Syria & 23 & 39 & 7.0 \\
\hline PI 211983 & Iran & 23 & 27 & 6.0 \\
\hline PI 370450 & Yugoslavia & 23 & 58 & 7.5 \\
\hline PI 255933 & Netherlands & 23 & 23 & 5.3 \\
\hline PI 339248 & Turkey & 23 & 21 & 5.3 \\
\hline PI 357830 & Yugoslavia & 23 & 53 & 7.0 \\
\hline PI 432877 & P.R. China & 23 & 19 & 6.5 \\
\hline PI 373918 & England & 23 & 21 & 6.3 \\
\hline PI 275410 & Netherlands & 23 & 13 & 6.3 \\
\hline PI 372898 & Netherlands & 23 & 26 & 5.3 \\
\hline PI 255937 & Netherlands & 24 & 23 & 6.0 \\
\hline PI 357843 & Yugoslavia & 24 & 26 & 8.0 \\
\hline Chicago Pickling & NSSL & 24 & 15 & 7.0 \\
\hline PI 357848 & Yugoslavia & 24 & 18 & 7.0 \\
\hline PI 204567 & Turkey & 24 & 28 & 6.7 \\
\hline Palmetto & NSSL & 24 & 40 & 6.3 \\
\hline PI 436672 & P.R. China & 24 & 18 & 6.3 \\
\hline PI 422171 & Czechoslovakia & 24 & 35 & 6.7 \\
\hline PI 481617 & Bhutan & 24 & 15 & 4.0 \\
\hline PI 214049 & India & 24 & 18 & 7.7 \\
\hline PI 222720 & Iran & 24 & 42 & 7.5 \\
\hline PI 390250 & Japan & 24 & 35 & 6.3 \\
\hline PI 432861 & P.R. China & 24 & 30 & 6.7 \\
\hline PI 169378 & Turkey & 24 & 22 & 7.7 \\
\hline PI 164743 & India & 24 & 15 & 6.7 \\
\hline PI 432875 & P.R. China & 24 & 34 & 7.0 \\
\hline PI 357855 & Yugoslavia & 24 & 11 & 7.3 \\
\hline PI 175688 & Turkey & 24 & 25 & 5.0 \\
\hline PI 175689 & Turkey & 24 & 19 & 5.7 \\
\hline PI 164173 & India & 24 & 14 & 6.7 \\
\hline PI 264227 & France & 25 & 31 & 6.3 \\
\hline PI 422174 & Czechoslovakia & 25 & 45 & 7.0 \\
\hline PI 267741 & Japan & 25 & 12 & 6.7 \\
\hline PI 432858 & P.R. China & 25 & 15 & 6.7 \\
\hline PI 174160 & Turkey & 25 & 22 & 7.3 \\
\hline PI 357833 & Yugoslavia & 25 & 41 & 8.7 \\
\hline PI 200815 & Burma & 25 & 36 & 5.7 \\
\hline PI 176521 & Turkey & 25 & 27 & 7.3 \\
\hline PI 169395 & Turkey & 25 & 31 & 8.3 \\
\hline PI 344442 & Iran & 25 & 22 & 6.3 \\
\hline Pixie & NSSL & 25 & 1 & 4.3 \\
\hline PI 175681 & Turkey & 25 & 11 & 7.5 \\
\hline PI 306179 & Poland & 25 & 34 & 7.7 \\
\hline PI 227013 & Iran & 25 & 47 & 7.3 \\
\hline PI 422169 & Czechoslovakia & 25 & 61 & 7.3 \\
\hline PI 357832 & Yugoslavia & 25 & 24 & 6.7 \\
\hline Wautoma & USDA-Wis & 25 & 31 & 5.0 \\
\hline PI 217946 & Pakistan & 25 & 31 & 7.0 \\
\hline PI 204569 & Turkey & 25 & 44 & 7.7 \\
\hline PI 432848 & P.R. China & 26 & 43 & 6.7 \\
\hline PI 263078 & USSR & 26 & 29 & 6.7 \\
\hline PI 432855 & P.R. China & 26 & 32 & 8.5 \\
\hline PI 264228 & France & 26 & 5 & 6.7 \\
\hline PI 177364 & Iraq & 26 & 30 & 6.7 \\
\hline PI 183127 & India & 26 & --- & 9.0 \\
\hline PI 206955 & Turkey & 26 & 19 & 7.3 \\
\hline PI 390241 & Japan & 26 & 26 & 4.0 \\
\hline PI 284699 & Sweden & 26 & 35 & 7.3 \\
\hline Delicatesse & NSSL & 26 & 8 & 6.7 \\
\hline PI 370447 & Yugoslavia & 26 & 6 & 6.5 \\
\hline PI 267742 & P.R. China & 26 & 31 & 7.3 \\
\hline PI 175950 & Turkey & 26 & 9 & 6.7 \\
\hline
\end{tabular}


loss in firmness were calculated from the data Average quality was the mean of the ratings for taste, seedcell wateriness, placental hollows, and carpel separation.

Damage traits (shriveling, fruit yellowing, and postharvest diseases) were rated subjectively on a $0-9$ scale $(0=$ none, $1-3=$ slight, 4 $6=$ moderate, $7-9=$ severe), and damage index was calculated as the mean of the three values. Percentage of loss in fruit weight and fruit firmness were calculated based on the formulas described for the germplasm screening study.

\section{Data analysis}

Germplasm screening study. The experiment was a randomized complete-block design with 761 cultigens and three replications. Data were analyzed using the GLM and CORR procedures of SAS (SAS Inst., Cary, N.C.).

Retest study. The experiment was performed using a factorial treatment arrangement in a randomized complete-block design with two replications of nine fruits per treatment combination [two harvest dates ( 1 and 13 July) $\times$ two storage locations $\times 20$ cultigens]. Data were analyzed using the GLM and CORR procedures of SAS (SAS Inst.).

\section{Results and Discussion}

Germplasm screening study. Differences among cultigens in percentage of fruit weight loss, percentage of fruit firmness loss, and degree of fruit shriveling during storage were highly significant. Cultigens were ranked based on the mean values for the percentage of fruit weight loss over the three replications (Table 1). Correlation analysis revealed that there were only moderate associations for percentage of fruit weight loss vs. fruit firmness loss $(r=0.60)$, percentage of fruit weight loss vs. shriveling rating $(r=0.44)$, and fruit firmness loss vs. shriveling rating ( $r=0.45)$ (Table 1$)$.

Cultigens with the lowest percentage of fruit weight loss $(<10 \%)$ were PI 172839, PI 344067, PI 264667, PI 171612, PI 339245, PI 220171, PI 279469, and PI 368550 (Table 1), and the cultigen with the highest percentage (70\%) was PI 357864. Cultigens with the lowest percentage of loss in fruit firmness
Table 1. Continued.

\begin{tabular}{|c|c|c|c|c|}
\hline Cultigen & Seed source ${ }^{y}$ & Wt loss $(\%)$ & $\begin{array}{c}\text { Firm. } \\
\text { loss }(\%)\end{array}$ & Shriveling $^{\mathrm{x}}$ \\
\hline PI 171608 & Turkey & 26 & 39 & 7.3 \\
\hline PI 224668 & Korea & 27 & 39 & 6.7 \\
\hline Earliest of All & NSSL & 27 & 19 & 5.7 \\
\hline Nappa 63 & NSSL & 27 & 2 & 6.5 \\
\hline MR 200 & NSSL & 27 & 21 & 6.0 \\
\hline PI 267745 & Brazil & 27 & 28 & 6.7 \\
\hline PI 169377 & Turkey & 27 & 14 & 7.0 \\
\hline PI 432862 & P.R. China & 27 & 26 & 6.0 \\
\hline PI 478366 & P.R. China & 27 & 36 & 7.0 \\
\hline PI 269481 & West Pakistan & 27 & 20 & 7.7 \\
\hline Robin & NSSL & 27 & 19 & 6.3 \\
\hline PI 458851 & USSR & 27 & 31 & 7.7 \\
\hline PI 390244 & Japan & 27 & 29 & 8.0 \\
\hline PI 137845 & Iran & 27 & 32 & 6.3 \\
\hline PI 263046 & USSR & 27 & 25 & 6.0 \\
\hline PI 211986 & Iran & 28 & 18 & 7.0 \\
\hline PI 390239 & Japan & 28 & 38 & 7.0 \\
\hline PI 419041 & P.R. China & 28 & 29 & 8.0 \\
\hline PI 176523 & Turkey & 28 & 47 & 7.3 \\
\hline PI 357869 & Yugoslavia & 28 & 33 & 7.3 \\
\hline PI 176525 & Turkey & 28 & 13 & 7.7 \\
\hline PI 212985 & India & 28 & 28 & 8.0 \\
\hline PI 432886 & P.R. China & 28 & 36 & 6.7 \\
\hline Sunny South & NSSL & 28 & 12 & 5.7 \\
\hline PI 372587 & Netherlands & 28 & 26 & 6.0 \\
\hline PI 261609 & Spain & 28 & 26 & 6.3 \\
\hline PI 163221 & India & 28 & 45 & 6.5 \\
\hline PI 379283 & Yugoslavia & 28 & 0 & 7.0 \\
\hline PI 372893 & Netherlands & 29 & 37 & 7.0 \\
\hline PI 422167 & Czechoslovakia & 29 & 21 & 7.0 \\
\hline PI 432851 & P.R. China & 29 & 52 & 8.0 \\
\hline PI 105263 & Turkey & 29 & 25 & 6.7 \\
\hline Staygreen & NSSL & 29 & 10 & 5.0 \\
\hline PI 357842 & Yugoslavia & 29 & 42 & 6.0 \\
\hline PI 228344 & Iran & 29 & 24 & 6.3 \\
\hline PI 296120 & Egypt & 30 & 31 & 7.0 \\
\hline PI 255938 & Netherlands & 30 & 24 & 8.0 \\
\hline Check-SMR 58 & Check & 30 & 22 & 7.7 \\
\hline PI 176524 & Turkey & 30 & 10 & 6.0 \\
\hline PI 169319 & Turkey & 30 & 5 & 6.0 \\
\hline PI 137851 & Iran & 30 & 12 & 5.7 \\
\hline PI 220860 & Korea & 31 & 17 & 6.0 \\
\hline PI 458846 & USSR & 31 & 33 & 7.0 \\
\hline PI 422192 & Czechoslovakia & 31 & 19 & 6.7 \\
\hline PI 175692 & Turkey & 31 & 42 & 7.0 \\
\hline PI 271328 & India & 31 & 26 & 7.7 \\
\hline PI 267744 & P.R. China & 31 & 37 & 6.3 \\
\hline PI 164284 & India & 31 & 19 & 7.7 \\
\hline PI 255935 & Netherlands & 31 & 20 & 5.7 \\
\hline PI 379280 & Yugoslavia & 31 & 65 & 8.0 \\
\hline PI 419135 & P.R. China & 31 & 47 & 7.3 \\
\hline PI 169328 & Turkey & 32 & 41 & 8.3 \\
\hline
\end{tabular}

Table 1. Continued.

\begin{tabular}{|c|c|c|c|c|}
\hline Cultigen & Seed source ${ }^{y}$ & Wt loss $(\%)$ & $\begin{array}{c}\text { Firm. } \\
\text { loss }(\%)\end{array}$ & Shriveling $^{\mathrm{x}}$ \\
\hline PI 390264 & Japan & 32 & 40 & 6.7 \\
\hline PI 357856 & Yugoslavia & 32 & 62 & 8.5 \\
\hline PI 279466 & Japan & 32 & 27 & 7.5 \\
\hline PI 265887 & Netherlands & 32 & 56 & 7.0 \\
\hline PI 169384 & Turkey & 32 & 45 & 7.3 \\
\hline PI 432871 & P.R. China & 33 & 65 & 7.0 \\
\hline PI 220169 & Afghanistan & 33 & 27 & 7.0 \\
\hline PI 390260 & Japan & 33 & 42 & 7.3 \\
\hline PI 212896 & India & 33 & --- & 5.0 \\
\hline PI 390261 & Japan & 33 & 21 & 7.0 \\
\hline PI 483344 & Korea & 33 & 66 & 8.0 \\
\hline PI 339243 & Turkey & 33 & 24 & 8.3 \\
\hline PI 422200 & Czechoslovakia & 33 & 25 & 7.0 \\
\hline PI 169334 & Turkey & 33 & 47 & 7.7 \\
\hline PI 164951 & Turkey & 33 & 41 & 7.7 \\
\hline PI 227235 & Iran & 33 & 21 & 6.3 \\
\hline PI 173893 & India & 34 & 31 & 8.0 \\
\hline PI 432864 & P.R. China & 34 & 48 & 8.3 \\
\hline PI 368560 & Yugoslavia & 34 & 16 & 6.7 \\
\hline PI 169350 & Turkey & 34 & 41 & 7.7 \\
\hline PI 279467 & Japan & 34 & 23 & 7.7 \\
\hline PI 169352 & Turkey & 35 & 44 & 7.3 \\
\hline PI 169390 & Turkey & 35 & 50 & 6.7 \\
\hline PI 357858 & Yugoslavia & 35 & 39 & 6.7 \\
\hline PI 368552 & Yugoslavia & 35 & 30 & 7.7 \\
\hline PI 368559 & Yugoslavia & 35 & --- & 8.0 \\
\hline PI 171613 & Turkey & 35 & 8 & 5.3 \\
\hline PI 422182 & Czechoslovakia & 36 & 13 & 4.3 \\
\hline Check-M 27 & Check & 36 & 46 & 7.7 \\
\hline PI 432882 & P.R. China & 37 & --- & 9.0 \\
\hline PI 205181 & Turkey & 37 & 37 & 8.5 \\
\hline PI 288991 & Hungary & 38 & 41 & 6.7 \\
\hline PI 458856 & USSR & 38 & 60 & 6.3 \\
\hline PI 285608 & Poland & 38 & 22 & 8.0 \\
\hline PI 342951 & Denmark & 38 & 39 & 7.7 \\
\hline PI 202801 & Syria & 38 & 34 & 8.0 \\
\hline PI 165509 & India & 38 & 24 & 8.3 \\
\hline PI 368557 & Yugoslavia & 38 & 6 & 8.0 \\
\hline PI 283901 & Czechoslovakia & 39 & 30 & 6.0 \\
\hline PI 197085 & India & 39 & 58 & 8.0 \\
\hline PI 368555 & Yugoslavia & 39 & 47 & 8.5 \\
\hline PI 390267 & Japan & 40 & 44 & 6.0 \\
\hline PI 223841 & Philippines & 40 & 37 & 7.3 \\
\hline PI 379281 & Yugoslavia & 40 & 62 & 8.0 \\
\hline PI 436608 & P.R. China & 41 & 30 & 5.5 \\
\hline PI 271326 & India & 41 & 22 & 8.0 \\
\hline PI 222244 & Iran & 41 & 31 & 6.3 \\
\hline PI 422179 & Czechoslovakia & 41 & 28 & 6.0 \\
\hline PI 167043 & India & 42 & 46 & 6.7 \\
\hline PI 169382 & Turkey & 42 & 62 & 7.7 \\
\hline PI 176519 & Turkey & 42 & 41 & 6.0 \\
\hline PI 432896 & P.R. China & 43 & 67 & 7.7 \\
\hline
\end{tabular}


Table 1. Continued.

\begin{tabular}{lcccc}
\hline & & & & \\
Cultigen & Seed source & Wt loss $(\%)$ & loss $(\%)$ & Shriveling $^{\mathrm{x}}$ \\
\hline PI 220791 & Afghanistan & 43 & 39 & 7.3 \\
PI 118279 & Brazil & 43 & 59 & 8.0 \\
GiantWtArnstadt & NSSL & 44 & 58 & 9.0 \\
PI 171607 & Turkey & 44 & 44 & 7.0 \\
PI 271327 & India & 44 & 75 & 6.7 \\
Davis Perfect & NSSL & 46 & 61 & 7.0 \\
Stono & NSSL & 46 & --- & 9.0 \\
PI 167198 & Turkey & 46 & 41 & 6.0 \\
PI 390251 & Japan & 46 & 26 & 6.0 \\
PI 391570 & P.R. China & 47 & 63 & 8.0 \\
PI 288990 & Hungary & 47 & 43 & 6.3 \\
PI 176522 & Turkey & 47 & 47 & 7.3 \\
PI 222986 & Iran & 48 & 65 & 8.3 \\
Redlands & Australia & 48 & 38 & 8.3 \\
PI 432863 & P.R. China & 50 & 61 & 8.3 \\
PI 197088 & India & 50 & 59 & 8.0 \\
Magnolia & NSSL & 51 & 58 & 8.5 \\
Check-Wautoma & Check & 51 & 50 & 8.5 \\
PI 285610 & Poland & 52 & 48 & 7.3 \\
PI 357838 & Yugoslavia & 52 & 48 & 8.0 \\
PI 163223 & India & 52 & 54 & 8.7 \\
PI 422184 & Czechoslovakia & 53 & 7 & 8.5 \\
PI 339244 & Turkey & 53 & 77 & 8.0 \\
PI 432885 & P.R. China & 55 & 11 & 6.5 \\
PI 255934 & Netherlands & 56 & 73 & 7.7 \\
PI 432874 & P.R. China & 58 & --- & 9.0 \\
PI 368553 & Yugoslavia & 60 & 100 & 9.0 \\
& & & &
\end{tabular}

Table 1. Continued.

\begin{tabular}{lcccc}
\hline \hline Cultigen & Seed source & Wt loss $(\%)$ & $\begin{array}{c}\text { Firm. } \\
\text { loss }(\%)\end{array}$ & Shriveling $^{\mathrm{x}}$ \\
\hline PI 357864 & Yugoslavia & 70 & --- & 9.0 \\
LSD (5\%) & 21 & 32 & 1.9 \\
Mean & 20 & 20 & 6.2 \\
CV (\%) & 64 & 101 & 19 \\
Range & 122 & 113 & 7 \\
Range/LSD & 6 & 4 & 4
\end{tabular}

Correlations

$r$ (\% weight lost vs. $\%$ firmness lost $)=0.60^{* *}$

$r(\%$ weight lost vs. shriveling rating $)=0.44^{* *}$

$r$ (\% firmness lost vs. shriveling rating $)=0.45^{* *}$

$r$ (keeping rating vs. shriveling rating) $=-0.50^{* *}$

$r$ (keeping rating vs. $\%$ weight loss $)=-0.85^{*}$

$r$ (keeping rating vs. \% firmness loss $)=-0.94^{* *}$

$\overline{{ }^{2}}$ Data are means for three replications of three fruits each. Cultigens listed were used as the male parent to produce a hybrid with Gy 14 (with the exception of those listed as checks, which were the actual cultigens). Cultigens designated with a PI number came from the USDA germplasm collection in Ames, Iowa. The NSSL seed source is the National Seed Storage Laboratory in Fort Collins, Colo.

${ }^{y}$ Some countries listed now no longer exist as political units (Czechoslovakia, USSR, Yugoslavia).

${ }^{x}$ Rated from 0 to $9(0=$ no shriveling, $1-3=$ slight shriveling and discoloring, 4-6 = moderate shriveling and discoloring, 7-9 = severe shriveling and discoloring).

wegative values are due to sampling error.

${ }^{* *}$ Significant at $P=0.01$.

Table 2. Mean keeping ability of 20 cucumber cultigens over two locations after storage for 0 and $14 \mathrm{~d} .^{\mathrm{z}}$

\begin{tabular}{|c|c|c|c|c|c|c|c|c|c|c|c|c|}
\hline Cultigen & $\begin{array}{c}\text { Seed } \\
\text { source }\end{array}$ & $\begin{array}{c}\text { Avg } \\
\text { quality }\end{array}$ & $\begin{array}{l}\text { Damage } \\
\text { index }^{\mathrm{x}}\end{array}$ & $\begin{array}{l}\text { Wt loss } \\
(\%)\end{array}$ & $\begin{array}{l}\text { Firm. } \\
\text { loss }(\%)\end{array}$ & $\begin{array}{l}\text { Fruit } \\
\text { Taste }^{\mathrm{u}}\end{array}$ & $\begin{array}{l}\text { Seed- } \\
\text { cell }^{\mathrm{w}}\end{array}$ & $\begin{array}{c}\text { Plac. } \\
\text { holloww }\end{array}$ & $\begin{array}{l}\text { Carpel sep. } \\
\text { rating }^{\mathrm{w}}\end{array}$ & Shriveling & $\begin{array}{l}\text { Fruit } \\
\text { yellow } \\
\text { rating }\end{array}$ & $\begin{array}{c}\text { Disease } \\
\text { rating }^{v}\end{array}$ \\
\hline Control (0 d storage) & & 8.5 & $\mathbf{0 . 0}$ & $\mathbf{0}$ & $\mathbf{0}$ & 8.0 & 9.0 & 8.0 & 9.0 & 0.0 & 0.0 & 0.0 \\
\hline Calypso & Petoseed & 6.5 & 1.8 & 17 & 12 & 4.0 & 4.0 & 8.8 & 9.0 & 2.5 & 3.5 & 0.0 \\
\hline Regal & N.C. State Univ. & 6.2 & 1.5 & -8 & 8 & 3.8 & 3.3 & 8.6 & 9.0 & 1.9 & 2.9 & 0.1 \\
\hline Gy 14 x PI422177 & N.C. State Univ. & 6.1 & 1.5 & 15 & 10 & 3.7 & 3.4 & 8.6 & 8.7 & 2.7 & 2.3 & 0.7 \\
\hline Wautoma & USDA-Wis. & 6.0 & 1.6 & 6 & 5 & 3.2 & 3.0 & 9.0 & 9.0 & 1.8 & 3.2 & 0.0 \\
\hline Gy $14 \times$ Producer & N.C. State Univ. & 6.0 & 2.0 & 16 & 12 & 4.2 & 2.8 & 8.7 & 8.5 & 2.2 & 4.0 & 0.0 \\
\hline Gy 14 x PI255934 & N.C. State Univ. & 6.0 & 1.6 & 22 & 13 & 2.6 & 3.5 & 9.0 & 9.0 & 3.6 & 2.9 & 0.4 \\
\hline PI 339244 & Turkey & 6.0 & 1.5 & 30 & 27 & 3.0 & 3.0 & 9.0 & 9.0 & 3.5 & 3.0 & 0.0 \\
\hline Gy 14 x PI339244 & N.C. State Univ. & 6.0 & 3.1 & -5 & 10 & 3.0 & 3.3 & 8.8 & 8.9 & 2.9 & 5.4 & 0.8 \\
\hline Gy 14 x PI 211962 & N.C. State Univ. & 6.0 & 2.4 & 8 & 11 & 3.7 & 3.4 & 8.1 & 8.6 & 2.1 & 4.1 & 0.7 \\
\hline Sumter & Asgrow Seed & 5.9 & 2.4 & 9 & 15 & 2.7 & 2.9 & 9.0 & 9.0 & 3.9 & 4.1 & 0.6 \\
\hline Dasher II & Petoseed & 5.9 & 0.6 & 26 & 10 & 3.3 & 3.0 & 8.5 & 8.8 & 1.1 & 1.1 & 0.1 \\
\hline Marketmore 76 & Asgrow Seed & 5.9 & 0.9 & -5 & -1 & 2.8 & 2.8 & 9.0 & 9.0 & 1.8 & 1.8 & 0.0 \\
\hline Gy 14 x PI279469 & N.C. State Univ. & 5.9 & 3.9 & 33 & 21 & 3.2 & 3.0 & 8.4 & 8.8 & 4.6 & 6.6 & 1.2 \\
\hline Poinsett 76 & Petoseed & 5.8 & 0.9 & -8 & 9 & 3.5 & 3.2 & 8.3 & 8.2 & 1.7 & 1.8 & 0.0 \\
\hline Gy 14 x PI321006 & N.C. State Univ. & 5.8 & 1.4 & -6 & 6 & 3.6 & 3.9 & 7.6 & 8.0 & 2.4 & 2.7 & 0.0 \\
\hline PI 220171 & Afghanistan & 5.8 & 1.8 & --- & --- & 2.5 & 2.5 & 9.0 & 9.0 & 2.0 & 3.5 & 0.0 \\
\hline PI 422177 & Czechoslovakia & 5.8 & 2.4 & 16 & 4 & 2.8 & 2.6 & 9.0 & 8.6 & 3.0 & 3.8 & 1.0 \\
\hline Gy 14 x PI220171 & N.C. State Univ. & 5.7 & 2.3 & 10 & 18 & 2.8 & 2.9 & 8.5 & 8.5 & 3.0 & 4.0 & 0.6 \\
\hline Wis. SMR 18 & Northrup King & 5.6 & 2.9 & 10 & 9 & 3.4 & 3.3 & 7.8 & 8.1 & 3.1 & 5.9 & 0.0 \\
\hline Producer & NSSL & 5.1 & 3.3 & 2 & 10 & 3.0 & 2.1 & 7.1 & 8.0 & 3.3 & 5.6 & 1.0 \\
\hline \multicolumn{13}{|c|}{ Statistics } \\
\hline $\operatorname{LSD}(5 \%)$ & & NS & 1.0 & NS & NS & NS & NS & NS & NS & 1.2 & 1.3 & NS \\
\hline Mean & & 5.9 & 2.0 & 8.6 & 10.5 & 3.3 & 3.1 & 8.5 & 8.6 & 2.6 & 3.7 & 0.4 \\
\hline $\mathrm{CV}(\%)$ & & 10 & 59 & 345 & 125 & 30 & 34 & 16 & 11 & 45 & 37 & 345 \\
\hline \multicolumn{13}{|c|}{ Means for harvest date (d) } \\
\hline $7 \mathrm{~d}$ & & 7.3 & 1.5 & -4.7 & 3.0 & 6.7 & 5.6 & 8.5 & 8.5 & 1.2 & 2.8 & 0.3 \\
\hline $14 \mathrm{~d}$ & & 5.9 & 2.0 & 8.6 & 10.5 & 3.3 & 3.1 & 8.5 & 8.6 & 2.6 & 3.7 & 0.4 \\
\hline \multicolumn{13}{|c|}{ Location (14 d only) } \\
\hline Shed & & 5.8 & 2.0 & 12.3 & 14.4 & 3.1 & 3.1 & 8.4 & 8.6 & 3.0 & 3.5 & 0.5 \\
\hline $\mathrm{Lab}$ & & 6.0 & 2.0 & 6.7 & 6.8 & 3.5 & 3.1 & 8.7 & 8.7 & 2.4 & 3.8 & 0.2 \\
\hline
\end{tabular}

${ }^{2}$ Data are means for two replications, two harvests, two locations, and six fruits.

${ }^{y}$ Mean of ratings for taste, seedcell, placental hollows, and carpel separation.

${ }^{x}$ Mean of ratings for shriveling, fruit yellowing, and fruit disease.

wTraits rated subjectively on a 1 to 9 scale $(1-3=$ poor, $4-6=$ intermediate, $7-9=$ excellent).

'Traits rated subjectively on a 0 to 9 scale $(0=$ none, $1-3=$ slight, $4-6=$ moderate, $7-9=$ severe $)$.

${ }^{\mathrm{N}}$ Nonsignificant. 
were PI 379284, PI 339241, PI 414159, PI 422177, 'Regal' (check), PI 109483, Addis (check), PI 285603, PI 257486, and 'Calypso' (check), and the cultigen with the highest percentage of loss was PI 368553. The cultigens with the least degree of fruit shriveling during storage were 'Dasher II' (check), 'Sprint 440' (check), 'Texas Long', PI 390255, PI 432870, 'Pacer', PI 419078, PI 390247, PI 321011, and PI 414158, and those with the greatest degree of fruit shriveling were PI 368553, PI 183127, PI 432882, 'Stono', PI 432874, and PI 357864.

Retest study. No significant differences were detected between the two harvest dates and the two storage locations for the 20 cultigens tested in the study (Table 2). However, differences among cultigens were greater at 14 than at $7 \mathrm{~d}$ after harvest. Therefore, data are reported as means over two locations at $14 \mathrm{~d}$ after harvest.

The traits of greatest value in distinguishing keeping ability were ratings for shriveling and fruit yellowing, based on their low $\mathrm{CV}$ and high range/LSD (Table 2). Differences in all other traits measured were inconsistent or were not statistically significant. Cultigens with the best keeping ability (based on damage index) were all slicers: 'Dasher II', 'Marketmore 76', and 'Poinsett 76' (Table 2). The worst cultigens were 'Producer' (an obsolete cultivar) and 'Wis. SMR 18' (a black-spined cultivar).

In general, the tough skin of modern slicing cultigens and the white-spine trait of most new cultigens contributed to superior keeping ability. However, there were differences in keeping ability among the thin-skinned, whitespined cultigens (Table 2). 'Regal' was one of the best and 'Producer' was one of the worst for keeping ability. These two cultigens would make good check lines (positive and negative checks) for future tests of keeping ability among thin-skinned cultigens.

Other traits were too variable to provide significant differences among cultigens. Different sets of fruits had to be measured after 0,7 , and $14 \mathrm{~d}$ of storage, since the fruit were cut for evaluation of seedcell wateriness, placental hollow, carpel separation, and taste. Future studies could be done using the same fruits if seedcell wateriness, placental hollows, carpel separation, and taste were not evaluated. Since the cultigens did not differ for those quality traits, they could be ignored in favor of obtaining better data for weight loss. Negative values for percentage of loss of fruit weight and firmness were a result of sampling error (Table 2). If a nondestructive test were conducted, firmness after $14 \mathrm{~d}$ would have to be used instead of percentage of loss in firmness, since no initial measurement for fruit firmness can be made (to calculate the percentage of loss in firmness) without damaging the fruits.

Differences between the cultigens per se and their hybrids were nonsignificant for shriveling and fruit yellowing of PI 220171 and for fruit shriveling of PI 422177. However, differences for fruit yellowing between the hybrids and cultigens per se for PI 422177 were significant (Table 2).

Future studies should examine more closely the association between skin toughness, spine color, and keeping ability. Keeping ability should be routinely measured on potential new cultivars by measuring weight loss, firmness, degree of shriveling, and fruit yellowing after storage for $14 \mathrm{~d}$ under uniform conditions. Check lines could include 'Marketmore 76', 'Regal', 'Wis. SMR 18', and 'Producer'.

\section{Literature Cited}

Bakr, A.A. and R.A. Gawish. 1993. Technological aspects of keeping and pickling qualities of cucumbers as influenced by fertilizers. Plant Foods for Human Nutr. 44:17-28.

Biale, J.B. and R.E. Young. 1981. Respiration and ripening in fruit-Retrospect and prospect, $\mathrm{p} .1-$ 39. In: J. Friend and M.J.C. Rhodes (eds.). Recent advances in the biochemistry of fruit and vegetables. Academic, New York.

Buescher, R.W. 1986. Controlled atmosphere storage of pickling cucumbers. Annu. Mtg., Pickling Cucumber Improvement Comm., Pickle Packers Intl., College Station, Texas.

Hughes, G.R., C.W. Averre, and K.A. Sorensen. 1983. Growing pickling cucumbers in North Carolina. North Carolina Agr. Ext. Serv. Bul. No. AG-315.

Hutchins, A.E. 1940. Inheritance in the cucumber. J. Agr. Res. 60:117-128

Janse, J. and G.W.H. Welles. 1984. Effects of energy saving measures on keeping quality of tomato and cucumber fruits. Acta Hort. 163:261-269.

Kays, S.J. 1991. Postharvest physiology of perishable plant products. Van Nostrand Reinhold, New York.
Lower, R.L. and M.D. Edwards. 1986. Cucumber breeding, p. 173-207. In: M.J. Bassett(ed.). Breeding vegetable crops. AVI, Westfield, Conn.

Miller, C.H. and T.C. Wehner. 1989. Cucumbers, p. 245-264. In: N.A.M. Eskin (ed.). Quality and preservation of vegetables. CRC Press, Boca Raton, Fla.

Navazio, J.P. and J.E. Staub. 1994. Effects of soil moisture, cultivar, and postharvest handling on pillowy fruit disorder in cucumber. J. Amer. Soc. Hort. Sci. 119:1234-1242.

SAS Institute. 1988. SAS/STAT user's guide, release 6.03 ed. SAS Inst., Cary, N.C.

Schultheis, J.R. 1990. Pickling cucumbers. North Carolina State Agr. Ext. Hort. Info. Leaflet No. 14-A

Shanan, S.A., M.K. Higazy, and M.M. Shera. 1978. The effect of thiabendazole, dowicide and flavorseal wax on the keeping quality of cucumber fruits. Egyptian J. Hort. 5(1):53-63.

Swallow, W.H. and T.C. Wehner. 1986. Optimum plot size for measurement of yield in multiple and once-over harvest trials of pickling and fresh market cucumbers. Euphytica 35:493-501.

Swallow, W.H. and T.C. Wehner. 1989. Optimum allocation of plots to years, seasons, locations, and replications, and its applications to onceover harvest cucumber trials. Euphytica 43:5968.

Thomas, R.S. and J.E. Staub. 1992. Water stress and storage environment affect pillowy fruit disorder in cucumber. J. Amer. Soc. Hort. Sci. 117:394-399.

Wehner, T.C. 1986. Efficiency of 3 single-harvest tests for evaluation of yield in pickling cucumber. Euphytica 35:493-501.

Wehner, T.C. 1987. Value of 12 season-location combinations for cucumber yield trials in North Carolina. Cucurbit Genet. Coop. Rpt. 6: 38-39.

Wehner, T.C. 1989. Breeding for improved yield in cucumber. Plant Breed. Rev. 6:323-359.

Wehner, T.C. and C.H. Miller. 1984. Efficiency of single-harvest methods for measurement of yield in fresh-market cucumbers. J. Amer. Soc. Hort. Sci. 109:659-664.

Wehner, T.C. and C.H. Miller. 1987. Optimum plant density for multiple-harvest yield of determinate cucumbers. Cucurbit Genet. Coop. Rpt. 10:29-30

Wehner, T.C. and R.R. Horton, Jr. 1986. Performance of cultivars of four different cucumber types for fresh-market use in North Carolina. Cucurbit Genet. Coop. Rpt. 9:53-54.

Wehner, T.C. and R.W. Robinson. 1991. A brief history of the development of cucumber cultivars in the U.S. Cucurbit Genet. Coop. Rpt. $14: 1-4$. 